\title{
A Large Cohort of Neurocysticercosis in Shandong Province, Eastern China, 1997-2015
}

\author{
Xiaodan Huang, Zhonglei Wang, Jingxuan Kou, Hongmei Liu, Dehua Mao, Zhenhua Yu, Xin Liu, \\ Peng Cheng, and Maoqing Gong
}

\begin{abstract}
Neurocysticercosis (NCC) has a wide spectrum of neurologic and psychiatric manifestations, including epileptic seizures, high intracranial pressure, cognitive dysfunction, and meningoencephalitis. This study presents the clinical diagnosis of 2539 NCC patients in Shandong Province, Eastern China, from 1997 to 2015. The diagnosis was based primarily on clinical features, neuroimaging, immunology, and electroencephalogramy studies. In all age groups, seizures were found to be the most common manifestation, followed by headaches, memory loss, and limb numbness, and disability. After antiparasitic treatment, computed tomography and magnetic resonance imaging showed that most of the lesions had been completely absorbed in $2106(82.95 \%)$ patients, most of the lesions had been absorbed and a small proportion was converted into calcifications in 433 $(17.05 \%)$ cases. Indirect hemagglutination (IHA), enzyme-linked immunosorbent assay (ELISA), and circulating antigen $(\mathrm{CAg})$ serum tests initially gave $76.45 \%, 86.37 \%$, and $80.66 \%$ positive results, respectively. After antiparasitic therapy, the positive rates of the IHA, ELISA, and CAg tests were $74.77 \%, 84.70 \%$, and $3.75 \%$, respectively, showing no significant difference in antibody levels $(p>0.05)$, but a significant difference in CAg levels $(p<0.01)$. The clinical antiparasitic treatment of NCC with three to four courses of a combination of albendazole and praziquantel achieved satisfactory therapeutic efficacy.
\end{abstract}

Keywords: neurocysticercosis, clinical features, diagnosis, albendazole, and praziquantel

\section{Introduction}

C YSTICERCOSIS Is A parasitic disease that usually occurs in domestic animals such as pigs and cattle and is caused by the larval cysticercus or metacestode stage of Taenia solium, also known as pork tapeworm. The larvae can lodge in various human tissues and organs. Neurocysticercosis (NCC) occurs when the cysticercus is located in the central nervous system of human brain. This disease tends to be most common in rural areas where sanitation and hygiene conditions are poor; raw or undercooked pork is eaten; and breeding livestock, especially pigs, have access to human feces (Coyle et al. 2012, Del Brutto 2012, Mwanjali et al. 2013). However, $\mathrm{NCC}$ is increasingly emerging in developed countries due to global immigration (Wandra et al. 2015). In addition, NCC has occurred in several African and Asian countries, including India, Indonesia, Thailand, Cambodia, Nepal, Philippines,
Myanmar, Vietnam, Korea, and China (Roman et al. 2000, Ndimubanzi et al. 2010, Sorvillo et al. 2011).

$\mathrm{NCC}$ is one of the most common parasitic infectious diseases in China, accounting for $60-90 \%$ of cysticercosis, and has a wide spectrum of neurologic and psychiatric manifestations, including epileptic seizures, high intracranial pressure, cognitive dysfunction, and meningoencephalitis. Since the 1980s, praziquantel and albendazole has been widely used in the clinical antiparasitic treatment of NCC and have achieved satisfactory therapeutic efficacy. Although this treatment is well established, the dosage and course of antiparasitic drugs for NCC remain to be optimized, and new research is constantly under way in China on how to use antiparasitic drugs rationally to achieve optimal effect (Su and Si 2002, Zhu et al. 2002). In this study, we performed a systematic review of the diagnosis and treatment of NCC in Shandong Province, China, from 1997 to 2015 to provide valuable data for the diagnosis and treatment of NCC.

World Health Organization Collaborating Centre for Lymphatic Filariasis and Taeniasis/Cysticercosis, Shandong Institute of Parasitic Diseases, Shandong First Medical University \& Shandong Academy of Medical Sciences, Jining, Shandong Province, China.

(C) Xiaodan Huang et al. 2019; Published by Mary Ann Liebert, Inc. This Open Access article is distributed under the terms of the Creative Commons Attribution Noncommercial License (http://creativecommons.org/licenses/by-nc/4.0/) which permits any noncommercial use, distribution, and reproduction in any medium, provided the original author(s) and the source are cited. 


\section{Materials and Methods}

Patients diagnosed with NCC admitted to the Parasitology Department at Shandong Institute of Parasitic Diseases (SIPD) in Shandong Province, China, between February 1997 and June 2015 was enrolled in this study. SIPD has been appointed by the WHO as the collaborating center for lymphatic filariasis and taeniasis/cysticercosis since 1997, and the institute has made great contributions to the prevention, diagnosis, and laboratory research of cysticercosis in China.

\section{Diagnosis of NCC}

The criteria (WS 381-2012) drafted by our center were used for diagnosis of clinical NCC in China (Zhen et al. 2012). The criteria include the following: (1) clinical symptoms such as epilepsy, high intracranial pressure, mental disorders, and so on, with other causes of brain damage excluded; (2) cysticercus cellulosae identified surgically by tableting, incubation tests, or histopathologic examination; (3) serum or cerebrospinal fluid positive by immunologic examination; (4) typical images by computed tomography (CT), magnetic resonance imaging (MRI) examination or electroencephalogram; and (5) history of travel to or residence in endemic areas and a history of tapeworms or contact with tapeworm-infected patients. Neuroimaging and immunological examination methods, including CT, MRI, indirect hemagglutination (IHA), enzyme-linked immunosorbent assay (ELISA), circulating antigen (CAg), and electroencephalogramy (EEG), can provide accurate diagnosis and evaluation of NCC, and these methods are described below.

\section{Neuroimaging diagnosis}

The CT image of a cysticercus in the active period is shown as a round low-density cyst with a diameter of $0.5-2 \mathrm{~cm}$, and the eccentric scolex can be seen; a larger cyst with a diameter of $2-12 \mathrm{~cm}$ is surrounded by a faint ring due to hyperplasia of fibrous tissue, and the scolex is difficult to see. In the degenerative death period, a cyst is shown as a low-density edematous area in one or several places, and there is a nodular or annular hyperintense area in the edematous region after enhancement. In the inactive period, the cyst is a round or elliptical calcification with a clearly defined edge, $2-4 \mathrm{~mm}$ in diameter, and there is no edema around it.

For the MRI examination, during the active period, cerebral parenchymal cysticercosis and intraventricular cysticercosis can be clearly distinguished. $T_{1}$-weighted imaging shows the cysticercus as a circular hypointense region and the scolex as a point-like or comma-like hyperintense region. $T_{2^{-}}$ weighted imaging shows a round hyperintense region, and the scolex appears as a punctate hypointense region; in the degenerative death period, the scolex is unclear, and the surrounding edema is obviously aggravated. Both $T_{1^{-}}$ weighted images and $T_{2}$-weighted images show large area of edema, and the thickness of the reinforcing ring is significantly increased. Calcifications sometimes occur after death, showing a long $T_{1}$ and short $T_{2}$; in the inactive period, MRI shows a point with no signal.

\section{Immunological diagnosis}

A total of $2 \mathrm{~mL}$ venous blood was collected from patients, and 1-2 $\mathrm{mL}$ cerebrospinal fluid was collected by lumbar puncture under sterile conditions for immunological diagnosis.

The IHA test was carried out as follows: (1) samples were diluted with a 96-well V-type plexiglass microhemagglutination plate, 8 wells per row, and $0.5 \%$ rabbit serum per well $(50 \mu \mathrm{L})$ was added. Then, the tested serum was removed from in the first well with a $0.025 \mathrm{~mL}$ metal dilution rod and diluted to the seventh well. The eighth well was a $0.5 \%$ rabbit serum control. One drop $(50 \mu \mathrm{L})$ of antigen-sensitized red blood cells was added to each well; the plate was then shaken for 2 min on a vibrating microshaker and placed at room temperature for $1 \mathrm{~h}$ to observe the results. The red blood cells diluted 1:8 in the serum was positive for $(++)$ agglutination, and the maximum dilution of serum with red blood cells was also positive $(++)$.

The ELISA assay was carried out as follows: a polystyrene reaction plate was used as a carrier, and 1:2000 diluted antigen was added to the wells, $0.2 \mathrm{~mL}$ per well, and the plate was placed in a $37^{\circ} \mathrm{C}$ incubator for $2 \mathrm{~h}$ and then transferred to a $4^{\circ} \mathrm{C}$ refrigerator overnight. The next day, the plate was washed 3 times (3-5 min each), and $0.2 \mathrm{~mL}$ patient serum diluted 1:50 was added; next, the samples were incubated at $37^{\circ} \mathrm{C}$ for $2 \mathrm{~h}$ in a refrigerator. Then, the plate was washed 3 times in the same way as above; $0.2 \mathrm{~mL}$ enzyme conjugate diluted 1:2000 was added. Subsequently, the plate was incubated at $37^{\circ} \mathrm{C}$ for $3 \mathrm{~h}$ and rinsed 3 times; $0.2 \mathrm{~mL}$ substrate solution was added, followed $30 \mathrm{~min}$ later by stop solution. The absorbance $A$ value was measured by an enzyme labeling instrument at $450 \mathrm{~nm}$ (reference wavelength at $630 \mathrm{~nm}$ ). A tested serum $A$ value $\geq 1.5$ times the average $A$ value of the healthy control serum was considered positive.

$\mathrm{CAg}$ in the serum and cerebrospinal fluid of patients with cysticercosis was detected by the double antibody sandwich method. The $A_{450}$ of each well was determined by an enzymelabeled meter. The test was positive when the $A$ value of the sample well was 2.1 times higher than the $A$ value of the negative control well, and if the $A$ value of the negative control well was $<0.03$, then it was calculated by 0.03 .

\section{Electrophysiology diagnosis}

The EEG test was performed using a 16-lead EEG machine (Shenkang Technology Corporation NDWZ, China). The silver electrode was placed according to the international 10/ 20 system. Single and bipolar lead traces were performed in awake, quiet, and sitting status. The EEG results were judged by reference to Feng Yingkun's clinical electroencephalography (Feng 1980).

\section{Therapeutic regimen}

After admission, all patients were treated with pumpkin seeds and betel nuts. Patients with high intracranial pressure were treated with mannitol and dexamethasone to reduce intracranial pressure, and patients with frequent epilepsy were given antiepileptic treatment. Patients with enlarged ventricles and intraventricular cysticercosis were treated with lateral ventricle-peritoneal shunt and surgical excision. After the clinical symptoms were significantly improved, antiparasitic treatment was performed. All hospitalized patients were treated with anticysticercosis drug, albendazole $20 \mathrm{mg} / \mathrm{kg} / \mathrm{d}$, and praziquantel $30 \mathrm{mg} / \mathrm{kg} / \mathrm{d}$ for 12 days in the first course. Afterward, praziquantel was increased to 
$50 \mathrm{mg} / \mathrm{kg} / \mathrm{d}$ for 12 days in the second course, and praziquantel $50 \mathrm{mg} / \mathrm{kg} / \mathrm{d}$ for 12 days in the third course, with an intermittence of 2-3 months. The antiparasitic treatment generally includes a total of three or four courses according to the conditions of patients. In the process, mannitol, dexamethasone, and brain protective agent were also adopted when marked evidence of raised intracranial pressure was noted.

\section{Efficacy evaluation}

The criteria for evaluating the curative effect were as follows: (1) cure: the clinical symptoms and signs disappeared, the imaging examination showed no lesions, and the enlarged ventricles returned to normal size morphologically; (2) markedly effective: the clinical symptoms and signs disappeared or obviously improved, most of the cystic lesions (75-80\%) disappeared, and the remaining (20-25\%) turned into calcification; and (3) invalid: there was no significant improvement in clinical symptoms and signs or significant change in cystic lesions before and after treatment.

\section{Statistical analysis}

Pre and posttreatment analyses of neuroimaging, immunologic EEG results, and clinical features were carried out. Data analysis was performed using SPSS version 18.

\section{Results}

\section{General condition of the cohort}

A total of 2539 NCC patients were included in this study, with a mean enrolment age of $39.6 \pm 10.2$ years (range: $5-76$ years). Detailed information on the cohort is illustrated in Table 1. The highest incidence rate was found among the 4059-year age group (29.9 [27.6-32.2]), while the 1-19-year age group had the lowest incidence risk (10.1 [8.6-11.6]). The male to female ratio is $8: 3$. For the course of disease, the shortest duration was just 2 days, whereas the longest duration was up to 32 years. For the occupation, 1829 (72.04\%) patients were farmers. In addition, $53(2.09 \%)$ patients had a history of eating pork infected with $T$. solium tapeworm, and $394(15.52 \%)$ patients had defecated tapeworm proglottis in the hospital.

Table 1. Neurocysticercosis Incidence Rates By Sex, Residence, And Age

\begin{tabular}{lrl}
\hline Characteristic & $\begin{array}{c}\mathrm{n}(\%) \text { of } \\
\text { patients }\end{array}$ & $\begin{array}{c}\text { Incidence rate, } \\
\text { cases/1 million } \\
\text { population }(95 \% \text { CI) }\end{array}$ \\
\hline $\begin{array}{l}\text { Sex } \\
\text { Male }\end{array}$ & $1834(72.23)$ & $37.9(36.1-39.7)$ \\
$\quad$ Female & $705(27.77)$ & $14.9(14.0-15.8)$ \\
Residence & & \\
$\quad$ Rural & $1829(72.04)$ & $43.5(40.3-46.7)$ \\
$\quad$ Urban & $710(27.96)$ & $13.2(12.1-14.3)$ \\
Age group, years & & \\
$\quad$ 1-19 & $307(12.09)$ & $10.1(8.6-11.6)$ \\
$\quad 20-39$ & $1002(39.46)$ & $29.9(27.6-32.2)$ \\
$\quad 40-59$ & $927(36.51)$ & $43.0(39.1-46.9)$ \\
$>60$ & $303(11.94)$ & $28.5(25.3-31.7)$ \\
\hline
\end{tabular}

$\mathrm{CI}$, confidence interval.

\section{Neuroimaging diagnosis}

All 2539 patients with NCC underwent CT, and 469 atypical cases also underwent MRI. MRI showed that 341 cases showed significantly increased lesions compared with CT (mainly active lesions), and 128 cases showed less lesions than CT (mainly calcified lesions). The lesions were mainly located in the frontal, temporal, and parietal regions (Fig. 1). According to the classification of Enhui $\mathrm{Wu}$, patients with imaging findings were divided into nine types: (1) 781 cases of cerebral parenchyma cyst, (2) 213 cases of acute encephalitis, (3) 281 cases of patchy edema, (4) 203 cases of single cyst or multiple cysts, (5) 380 cases of nodular cyst, (6) 39 cases of meningitis, (7) 207 cases of multiple calcification, (8) 79 cases of ventricular cyst, and (9) 356 cases of mixed type. Among the patients, 341 cases showed significantly increased lesions (mainly active stage lesions), whereas 128 cases demonstrated reduced lesions (mainly calcified lesions) compared with the original CT (Wu 1995) (Table 2).

After 3-4 courses of antiparasitic treatment, 2170 patients subsequently underwent CT examination, and 369 received MRI again. The results showed that low-density lesions were completely absorbed in $2106(82.95 \%)$ patients. Lesions that were mostly absorbed were found in $433(17.05 \%)$ cases, among whom 72 hydrocephalus cases $(72 / 79,91.14 \%)$ returned to normal and 7 ventricle cases were significantly smaller than before surgery (Fig. 2).

\section{Immunological diagnosis}

Blood samples were collected from all patients with NCC (2539), and serum was isolated for IHA, ELISA, and CAg detection. In this study, 1941 (76.45\%) serum samples were IHA-positive, $2193(86.37 \%)$ were ELISA-positive, and 2048 (80.66\%) were CAg-positive. The dynamic changes in immunology were observed in 987 NCC patients after antiparasitic therapy. The positive rates of IHA, ELISA, and CAg were $74.77 \%, 84.70 \%$, and $3.75 \%$, respectively, showing no significant difference in the antibody $(p>0.05)$, but a significant difference in the CAg $(p<0.01)$. In addition, 569 patients provided cerebrospinal fluid for testing, 409 (71.88\%) samples were IHA-positive, $475(83.48 \%)$ were ELISA-positive, and 412 (72.41\%) were CAg-positive.

\section{EEG examination}

All patients with NCC received EEG examination. The results indicated that $915(36.04 \%)$ were normal, while 1624 $(63.96 \%)$ were abnormal. Among the abnormal cases, 679 $(41.81 \%)$ were mildly abnormal, $741(45.63 \%)$ were moderately abnormal, and $204(12.56 \%)$ were severely abnormal. After treatment, $1253 \mathrm{NCC}$ patients were rechecked by EEG, and the results showed that 938 cases $(74.86 \%)$ returned to normal, 251 cases $(20.03 \%)$ were obviously improved, and 64 cases were still mildly abnormal.

\section{Clinical features}

The clinical features are illustrated in Table 3. Seizures/ epilepsy (1427 cases) was found to be the most common feature, followed by headaches (894 cases), memory loss ( 239 cases), limb numbness and disability (229 cases), speech impairment (85 cases), blurred vision and diplopia ( 74 cases), and mental disorders (49 cases). Fundus examination 

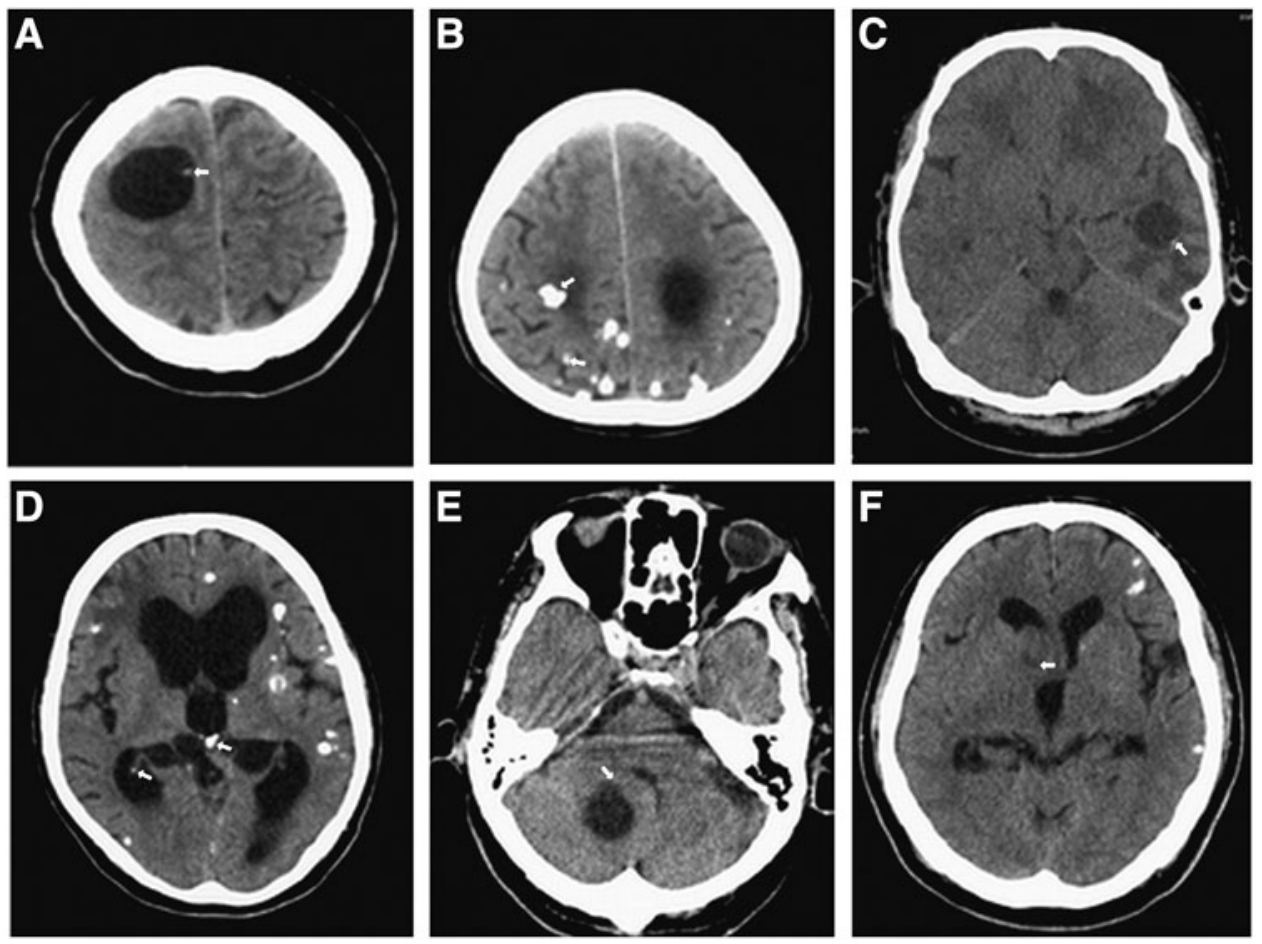

FIG. 1. Different sites of NCC. Pariental NCC (A, B), frontal NCC (C), temporal NCC (D), cerebellar NCC (E), and intraventricular NCC (F). NCC, neurocysticercosis. identified 102 cases of papilledema and 19 cases of papilloedema with hemorrhage. Cerebral spinal fluid examination identified 391 cases with intracranial pressure. Cysticercus was also found in subcutaneous nodules in 127 patients by surgical extraction.

After antiparasitic therapy, 1349 (94.53\%) patients with epilepsy had their seizures completely under control, and 78 $(5.47 \%)$ patients with epilepsy showed reduced seizure-onset frequency and duration. Of the 894 patients with headache, dizziness, nausea, and vomiting, 861 (96.31\%) recovered, and only $33(3.69 \%)$ still had symptoms when they caught a cold or felt tired. For patients with memory loss, 223 $(93.31 \%)$ returned to their preillness conditions, while 16 $(6.69 \%)$ had no significant improvement. All NCC patients with limb numbness and disability, speech impairment, blurred vision and diplopia, mental disorders, papilledema, papilledema with hemorrhage, and increased intracranial pressure returned to normal. For the 127 patients with sub-

TABle 2. The Types of NCC PATIENTS BY IMAGING Diagnosis

\begin{tabular}{|c|c|c|}
\hline Types & No. of NCC patients & $\%$ \\
\hline Cerebral parenchyma cyst & 781 & 30.76 \\
\hline Acute encephalitis & 213 & 8.39 \\
\hline Patchy edema & 281 & 11.07 \\
\hline Single cyst or multiple cysts & 203 & 8.00 \\
\hline Nodular cyst & 380 & 14.97 \\
\hline Meningitis & 39 & 1.54 \\
\hline Multiple calcification & 207 & 8.15 \\
\hline Ventricular cyst & 79 & 3.11 \\
\hline Mixed type & 356 & 14.02 \\
\hline Total & 2539 & \\
\hline
\end{tabular}

NCC, neurocysticercosis. cutaneous nodules, nodules disappeared after two courses of antiparasitic treatment.

\section{Adverse drug reactions}

During the first course of antiparasitic treatment, 744 (29.30\%) NCC patients had adverse drug reactions, mostly within a week, with the main manifestations of fever, headache, dizziness, nausea, vomiting, malaise, allergic reactions, seizures, numbness, and disability. Among them, 341 cases $(45.83 \%)$ had headache and dizziness, 386 cases $(51.88 \%)$ had fever, and some patients had both fever and headache. These patients were suspended to use antiparasitic drugs and given appropriate treatment in a timely manner. In the second course of treatment, only $91(12.31 \%)$ patients who were severely infected had adverse drug reactions, but the symptoms and severity were significantly reduced.

\section{Discussion}

Shandong Province used to be one of the most epidemic areas of cysticercosis in China. The infection rate of cysticercosis was $0.71 \%$ in Shandong province in 1986 (Cao and $\mathrm{Xu} 1995)$, and the prevalence of cysticercosis was reduced to $0.11 \%$ in 1997 (Liu et al. 2000). This study found that NCC patients were predominately middle-aged, more men than women, and the occupation was dominated by farmers. The main clinical manifestations of NCC included seizures, headache, dizziness, nausea, memory loss, and limb numbness. These clinical symptoms depend on the number of parasites, the location, the life stage of the cysticercus, the immune response of the host and so on (Carabin et al. 2011, Nash and Garcia 2011, Sierra et al. 2017). The evolution of cerebral cysticercus generally involves three stages: active, degenerative, and calcification stages. Their damage to brain tissue varies in different stages, and the harm is the greatest in 

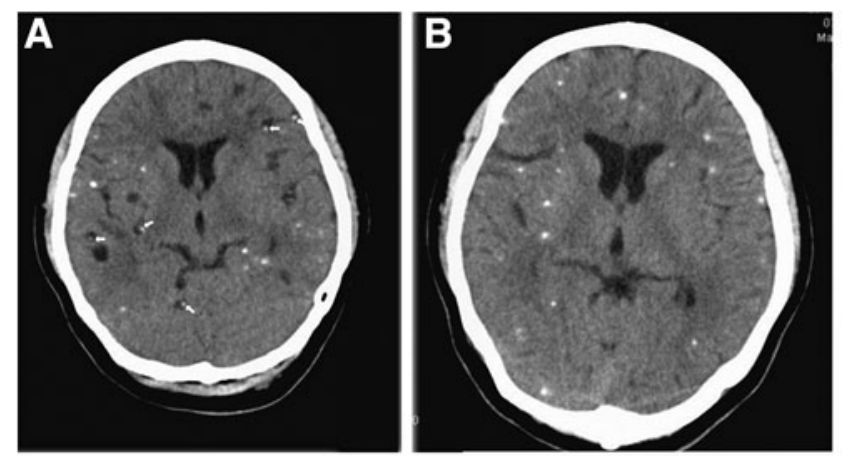

FIG. 2. Computed tomography image of NCC before treatment $(\mathbf{A})$ and 1 year after the antiparasitic treatment $(\mathbf{B})$.

the degenerative stage. During this period, cysticercus releases large amounts of allogeneic protein, which leads to cerebral edema, inflammation in brain tissues, fibrosis, the blockage of the cerebrospinal fluid pathway, resulting in increased intracranial pressure and even palsy, thus showing different symptoms in the clinic. In this study, after 3-4 courses of antiparasitic treatment, most of the lesions in NCC patients were absorbed, and only a small number were converted to calcification. The total effective rate was up to $100 \%$, which was similar to the report that $80 \%$ of the cerebral parenchyma cysticercosis were destroyed and absorbed without calcification after therapy (Wu et al. 2013). After the antiparasitic treatment, as the antigen produced by the cysticercus decreased or disappeared, the immune response was reduced, and with the organization and neovascularization, the function of brain gradually returned to normal; thus, clinical symptoms disappeared or relieved. The recovery of patients with clinical symptoms was a quite slow process, mainly due to the severity of infection, untimely treatment, and the formation of calcifications of the worm. Therefore, patients should avoid overwork and drinking in their daily lives to improve their quality of life and keep healthy.

Imaging examination is very important for the diagnosis and treatment of NCC, because it can be used not only to judge the location, the infection conditions, and the stage of cerebral cysticercus but also to identify low-density fresh lesions and high-density calcification. When the cysticercus is active, the cyst wall was thin and had not been surrounded by fibrous tissue, this was the best time for anticysticercus treatment. After taking medicine, the cyst wall was ruptured and cysticercus was decomposed and liquefied, which can be absorbed completely, and few calcifications were formed. In contrast, in the degeneration and death stage, cysticercus began to degenerate, cystic fluid became turbid, allogeneic protein entered into the cerebral tissue, the surrounding tissue was organized and inflammatory granuloma was formed, surrounded by fibrous tissue, and the cystic wall became thick, so it was difficult for medicine to act on the cysticercus. Then, the cysticercus gradually became fibrosis and calcified. The absorption of calcification was quite difficult, so some patients still had mild brain symptoms after treatment (Jia et al. 2003, Wang et al. 2008, Cangalaya et al. 2017, Singh et al. 2017). Therefore, once the diagnosis of NCC was confirmed, treatment should be taken promptly so that the lesions could be completely absorbed. For patients with calcified lesions, blood circulation, and brain function recovery measures should be performed to control the clinical symptoms, but cysticidal drug was not needed ( Jia et al. 2005, Nash et al. 2008).

Immunological examination is of great value in the diagnosis of NCC, but it is of little significance to the early diagnosis because the antibody does not appear until a few weeks after infection and lives in the serum for as long as 10 years (Feng and Shen 1989). The detection of CAg is not only diagnostically valuable in the early stage of disease but also useful as a reference for evaluating the curative effect of anticysticercosis treatment, since CAg is the discharge or excrement of cysticercus into the host body, which appears earlier than antibody and coexists with cysticercus. The amount of CAg is consistent with the degree of infection and disappears with the death of cysticercus (Li 1991, Montano et al. 2005). It was reported that the changes in clinical symptoms were consistent with the changes in serum $\mathrm{CAg}$, and the $\mathrm{CAg}$ of the patients decreased or disappeared significantly 30 days after the death of cysticercus (Zhai et al. 1993). The results of this study are consistent with the report above.

EEG is considered an index that reflects the function of cerebral cells. This modality is essentially different from CT and MRI anatomical imaging in the diagnosis of cerebral diseases, especially in providing valuable information in the early stage of various organic brain diseases. In this study, the abnormal rate of EEG was $63.96 \%$, mainly mild and

Table 3. Clinical Presentations of NCC Patients

\begin{tabular}{lccc}
\hline Features & No. of NCC & $\%$ & $95 \%$ CI \\
\hline Seizures/epilepsy & 1427 & 56.20 & $53.59-58.81$ \\
Simple partial & 963 & 67.48 & $61.25-73.71$ \\
Complex partial & 371 & 26.00 & $21.77-30.23$ \\
Generalized & 93 & 6.52 & $4.20-8.84$ \\
Headaches & 894 & 35.22 & $22.85-47.59$ \\
Memory loss & 239 & 9.41 & $5.67-13.15$ \\
Limb numbness and disability & 229 & 9.02 & $5.43-12.61$ \\
Speech impairment & 85 & 3.35 & $1.12-5.58$ \\
Blurred vision and diplopia & 74 & 2.95 & $0.95-4.95$ \\
Mental disorders & 49 & 1.93 & $0.44-3.42$ \\
Raised intracranial pressure & 391 & 15.40 & $10.71-20.09$ \\
Subcutaneous nodules & 127 & 5.00 & $2.89-7.11$ \\
\hline
\end{tabular}


moderate abnormal. After antiparasitic treatment, $1253 \mathrm{NCC}$ patients were reviewed, $94.89 \%$ returned to normal or significantly improved, and the metabolic neuronal function of brain cells gradually returned to normal, indicating that most of the brain damage caused by NCC can be recovered gradually. The change in EEG is basically consistent with the development of the disease. As the patient is cured or improved, the EEG gradually becomes normal or basically normal.

No effective drug for the treatment of NCC had been discovered before the 1980s in the world. Some domestic scholars have made pills with a variety of Chinese herbal medicines, such as GanWupulvis (Ge et al. 1991). However, these therapies usually lasted more than 1 year, and the treatment efficacy was uncertain. Since the 1980s, the advent of praziquantel and albendazole made a breakthrough in the treatment of NCC. Specific antiparasitic treatment used either praziquantel or albendazole alone, with dosages ranging from 10 to $60 \mathrm{mg} / \mathrm{k} / \mathrm{d}$, and the therapeutic result was not satisfactory (Ma et al. 1984, Sotelo et al. 1990, Botero et al. 1993, Garcia et al. 2004, Del Brutto et al. 2006). The present study designed an increasing dosage and prolonged course of treatment to ensure the effective time of the drug in vivo based on the site and mechanism of albendazole and praziquantel acting on NCC, the pharmacokinetics, blood, and cerebrospinal fluid concentration. Clinical observations have shown that some NCC patients were repeated infections, and the degree of infection and parasitic locations were different. Moreover, diapause phenomena occurred in the body, usually lasting 60-270 days. All of these brought difficulties to clinical treatment, and it was necessary to give patients multiple intermittent medications, based on the development of oncosphere to cysticercus. According to the developmental cycle of cerebral cysticercus, multiple courses of treatment were used with an interval of 2-3 months. Due to the complex antigen components, albendazole was first taken to deplete the glycogen in cysticercus and destroy the microenvironment of its survival so that cysticercus became inactive in the brain tissue. Then, praziquantel was taken to quickly kill cysticercus whose vitality had been greatly reduced, thereby reducing the insecticidal response in the treatment of praziquantel. In the course of treatment, the personalized scheme was adopted; that is, different doses of mannitol and corticosteroids were applied. Mannitol can increase plasma osmotic pressure and transfer water in the brain tissue into the blood vessels, thereby eliminating brain edema and reducing intracranial pressure. Corticosteroids are able to stabilize the blood-brain barrier, inhibit the immune response and stabilize the cell membrane structure to achieve dehydration and intracranial pressure reduction. In addition, both have the effect of scavenging free radicals in the brain, which is beneficial to protect brain cells. According to the patient's infection, after the second course of treatment, the dosage was gradually increased supplemented with blood circulation drugs and brain cell protection agents to promote lesion absorption and restore brain cell function. The combined therapy of the above two drugs was adopted in the present study, and the results demonstrated that the combination had a much higher parasiticidal efficacy against viable cysticercus compared with the monotherapy, which was in accordance with other studies (Garcia et al. 2014, 2016).
During and after antiparasitic treatment, patients may have adverse drug reactions, especially in the first week of taking medication. In this study, patients were given albendazole combined with liver protection, followed by praziquantel; the antiparasitic response rate was $29.30 \%$, which was significantly reduced compared with the previous therapy with praziquantel alone ( $\mathrm{Li}$ and $\mathrm{Li}$ 1998). It was advisable to adopt personalized medication plan based on the severity of infection and the size of the lesion. Medication should be gradually increased from a low dose to a full dose, and dehydrating agents and corticosteroids should be added from the beginning of treatment to reduce intracranial pressure and prevent adverse drug reactions. The incidence of sequelae in patients treated with dehydrating agent and corticosteroids was significantly lower than that in patients without corticosteroids ( $p<0.05)$ (Xu et al. 2002). Once fever, headache, dizziness, seizures, and other symptoms appear, drugs must be stopped promptly, and treatment should be conducted to the symptoms until they were alleviated. After the patient was discharged from the hospital, due to the dissolution and absorption of dead parasites, the toxins still stimulate the body, causing adverse drug reactions and sometimes lifethreatening. Therefore, guardianship for approximately 1 week was necessary to prevent the delayed adverse drug response.

\section{Acknowledgments}

This study was supported by grants from the National Natural Science Foundation of China (grant nos. 81672059 and 81871685), the Key Research and Development Program of Shandong Province (nos. 2018GSF118092 and 2018GSF118040), the Shandong Provincial Health Department (grant no. 2017WS102), and the Innovation Project of Shandong Academy of Medical Sciences.

\section{Author Disclosure Statement}

No conflicting financial interests exist.

\section{References}

Botero D, Uribe CS, Sanchez JL, Alzate T, et al. Short course albendazole treatment for neurocysticercosis in Columbia. Trans R Soc Trop Med Hyg 1993; 87:576-577.

Cangalaya C, Bustos JA, Calcina J, Vargas CA, et al. Radiological evolution of porcine neurocysticercosis after combined antiparasitic treatment with praziquantel and albendazole. PLoS Negl Trop Dis 2017; 11:e0005624.

Cao WC, Xu JF. Epidemiological survey on Taenia solium and cysticercus sac infection in Shandong Province, China. Chin J Parasit Dis Control 1995; 8:168-172.

Carabin H, Ndimubanzi PC, Budke CM, Nguyen H, et al. Clinical manifestations associated with neurocysticercosis: A systematic review. PLoS Neg1 Trop Dis 2011; 5:e1152.

Coyle CM, Mahanty S, Zunt JR, Wallin MT, et al. Neurocysticercosis: Neglected but not forgotten. PLoS Negl Trop Dis 2012; 6:e1500.

Del Brutto OH. Diagnostic criteria for neurocysticercosis, revisited. Pathog Glob Health 2012; 106:299-304.

Del Brutto OH, Roos KL, Coffey CS, Garcia HH. Meta-analysis: Cysticidal drugs for neurocysticercosis: Albendazole and praziquantel. Ann Intern Med 2006; 145:43-51. 
Feng XC, Shen YP. The detection of circulatng antigen of parasitic infection and its application. J Pathog Biol 1989; 2: 110-113.

Feng YK. Clinical Electroencephalography. Beijing: People's Health Publishing House, 1980:61-64.

Garcia HH, Gonzales I, Lescano AG, Bustos JA, et al. Efficacy of combined antiparasitic therapy with praziquantel and albendazole for neurocysticercosis: A double-blind, randomised controlled trial. Lancet Infect Dis 2014; 14:687-695.

Garcia HH, Lescano AG, Gonzales I, Bustos JA, et al. Cysticidal efficacy of combined treatment with praziquantel and albendazole for parenchymal brain cysticercosis. Clin Infect Dis 2016; 62:1375-1379.

Garcia HH, Pretell EJ, Gilman RH, Martinez SM, et al. A trial of antiparasitic treatment to reduce the rate of seizures due to cerebral cysticercosis. N Engl J Med 2004; 350:249-258.

Ge LY, He PJ, Cao YC, Wang ZH, et al. Histopathological observation on cysticercus cellulosae aftek treatment with ganwupulvis, a Chinese traditional medicine. J Pathog Biol 1991; 4:22-23.

Jia FJ, OY AM, Wu XY, Hu YX, et al. Change of CT image and clinical significance in cerebral cysticercosis treated by PQT and PQT with dose increasing method. Bull Dis Control Prev 2005; 20:7-9.

Jia FJ, Wu XY, Dai W, Sun GP, et al. Analysis of CT image and effect of anti-cysticercus therapy for 300 patients with cerebral cysticercosis. Chin J Schisto Control 2003; 15:282-284.

Li CM, Li CX. Clinical observation on 116 cerebral cysticercosis patients treated with big dosage of praziquantel. Chin J Int Med 1998; 37:474.

Li YH. Parasitology Immunology and Immunodiagnosis. Nanjing, China: Jiangsu Science and Technology Press, 1991:55.

Liu X, Deng XL, Zhao ZP, Wan GQ, et al. Epidemiological studies on taeniasis and cysticercosis in the middle part of Shandong province. Chin J Parasit Dis Control 2000; 3:34-36.

Ma Y, Zhu J, Liu Z. A clinical study on the treatment of cerebral cysticercosis with praziquantel. Natl Med J China 1984; 64:79-83.

Montano SM, Villaran MV, Ylquimiche L, et al. Neurocysticercosis: association between seizures, serology and brain CT in rural Peru. Neurology 2005; 65:229-233.

Mwanjali G, Kihamia C, Kakoko DV, Lekule F, et al. Prevalence and risk factors associated with human Taenia solium infections in Mbozi District, Mbeya Region, Tanzania. PLoS Negl Trop Dis 2013; 7:e2102.

Nash TE, Pretell EJ, Lescano AG, Bustos JA, et al. Perilesional brain oedema and seizure activity in patients with calcified neurocysticercosis: A prospective cohort and nested casecontrol study. Lancet Neurol 2008; 7:1099-1105.

Nash TE, Garcia HH. Diagnosis and treatment of neurocysticercosis. Nat Rev Neurol 2011; 7:584-594.

Ndimubanzi PC, Carabin H, Budke CM, Nguyen H, et al. A systematic review of the frequency of neurocyticercosis with a focus on people with epilepsy. PLoS Negl Trop Dis 2010; 4:e870.

Roman G, Sotelo J, Del Brutto O, Flisser A, et al. A proposal to declare neurocysticercosis an international reportable disease. Bull World Health Organ 2000; 78:399-406.

Sierra MM, Arroyo M, Torres MC, Cruz NR, et al. Extraparenchymal neurocysticercosis: Demographic, clinicoradiological and inflammatory features. PLoS Negl Trop Dis 2017; 11:e0005646.
Singh SK, Prasad KN, Singh AK, Gupta KK, et al. Adhesion molecules, chemokines and matrix metallo-proteinases response after albendazole and albendazole plus steroid therapy in swine neurocysticercosis. Exp Parasitol 2017; 182:1-8.

Sotelo J, Del Brutto OH, Penagos P, Escobedo F, et al. Comparison of therapeutic regimen of anticysticercal drugs for parenchymal brain cysticercosis. J Neurol 1990; 237:69-72.

Sorvillo F, Wilkins P, Shafir S, Eberhard M. Public health implications of cysticercosis acquired in the United States. Emerg Infect Dis 2011; 17:1-6.

Su RN, Si Q. Clinical observation on the efficacy and sideeffects of praziquantel treatment in 6480 cases of cysticercosis. Chin J Parasitol Parasit Dis 2002; 20:320.

Wandra T, Swastika K, Dharmawan NS, Purba IE, et al. The present situation and towards the prevention and control of neurocysticercosis on the tropical island, Bali, Indonesia. Parasit Vectors 2015; 8:148.

Wang ZL, Kou JX, Hu YX, Liu YL, et al. Clinical observation on the effect of combined use of praziquantel and albendazole on cerebral cysticercosis. China Trop Med 2008; 8:1873-1876.

Wu EH. Head CT Diagnostic. Beijing: People's Medical Publishing House, 1995:146.

Wu W, Jia FJ, Wang W, Huang Y, et al. Antiparasitic treatment of cerebral cysticercosis: Lessons and experiences from China. Parasitol Res 2013; 112:2879-2890.

Xu HX, Yin KX, Xu J, Liu YB. Study on measures to alleviate the sequelae of cerebral cysticercosis. Chin J Dis Control Prev 2002; 17:69-72.

Zhai CS, Wang YS, Shi ZL. Study on the detection of circulating antigen of patients with cysticercosis by ELISA. J Pathog Biol 1993; 6:16-18.

Zhen TM, Yang YJ, Li DJ, Ge LY, et al. Diagnosis of cysticercosis. Diagnostic criteria of the health industry in the People's Republic of China 2012; WS381:1-14.

Zhu SX, Diao WZ, He ZY. Clinical treatment of cerebral cysticercosis (analysis of 420 cases). Acta Acad Med Hebei 2002; 13:145-147.

Address correspondence to: Peng Cheng

World Health Organization Collaborating Centre for Lymphatic Filariasis and Taeniasis/Cysticercosis Shandong Institute of Parasitic Diseases Shandong First Medical University Shandong Academy of Medical Sciences Jining, Shandong 272033

China

E-mail: cpzuye@aliyun.com

Maoqing Gong

World Health Organization Collaborating Centre for Lymphatic Filariasis and Taeniasis/Cysticercosis Shandong Institute of Parasitic Diseases Shandong First Medical University Shandong Academy of Medical Sciences Jining, Shandong 272033

China

E-mail: gmq2005@163.com 